REVISTA EVIDENCIAÇÃO CONTÁBIL \& FINANÇAS

João Pessoa, v. 4, n. 3, p. 30-43, set./dez. 2016.

ISSN 2318-1001

DOI: 10.18405/recfin20160302

Disponível em: http://periodicos.ufpb.br/ojs2/index.php/recfin

\title{
CONTRIBUIÇÕES DOS INVESTIMENTOS EM BOLSAS STRICTO SENSU NA PRODUÇÃO ACADÊMICA BRASILEIRA ${ }^{1}$
}

\section{CONTRIBUTIONS OF INVESTMENT IN SCHOLARSHIPS STRICTO SENSU IN BRAZILIAN ACADEMIC PRODUCTION}

\author{
Khalil Gibran Martins Zeraik Abdalla ${ }^{2}$ \\ Doutorando em Administração pela Pontifícia Universidade Católica do Paraná (PUC-PR) \\ kzabdalla@gmail.com \\ Alysson Bryan Citadin \\ Graduado em Administração pela Pontifícia Universidade Católica do Paraná (PUC-PR) \\ alysson.citadin@solmarista.org.br \\ Luiz Pinheiro Junior \\ Doutorando em Administração pela Fundação Getúlio Vargas (FGV) de São Paulo (EAESP) \\ luizpinheirojunior@gmail.com
}

\section{RESUMO}

A informação é um recurso determinante dentro das organizações, visto sua capacidade de gerar inovações e melhores práticas gerenciais. É possível verificar por meio disso que são necessários investimentos financeiros para geração de informação e conhecimentos que contribuam com as organizações e sociedade. Neste sentido, o objetivo deste artigo é analisar a relação entre o investimento da Coordenadoria de Aperfeiçoamento de Pessoal de Nível Superior (CAPES) em bolsas acadêmicas stricto sensu com as publicações acadêmicas brasileiras, no período de 2000 a 2012. Para isso foi realizado um estudo exploratório e descritivo com análise estatística dos dados com as técnicas de regressão polinomial e dispersão utilizando base de dados secundários. Foi possível verificar evidências empíricas por meio dos coeficientes de determinações dos modelos estatísticos. Os resultados demostram que apesar dos dados da CAPES demonstrarem constante aumento no investimento em bolsas stricto sensu no Brasil no período de 2000 - 2012, não se verifica aumento proporcional da produção científica nacional no mesmo período. Bem como não contribuíram efetivamente para o aumento da representatividade do país no campo acadêmico perante a América Latina e ao Mundo. As considerações finais destacam os principais fatores identificados nas análises estatísticas que levam a tais conclusões. O estudo contribui para o campo teórico dos estudos que mensuram investimentos e inovação e também auxilia de forma aplicada aos gestores de recursos públicos.

\footnotetext{
${ }_{1}$ Artigo recebido em: 08/06/2016. Revisado por pares em: 21/08/2016. Reformulado em: 29/09/2016. Recomendado para publicação em: 29/09/2016 por Luiz Felipe de Araújo Pontes Girão (Editor Adjunto). Publicado em: 28/10/2016. Organização responsável pelo periódico: UFPB.

${ }^{2}$ Endereço: Rua Imaculada Conceição, 1155, Bloco Acadêmico, 1º Andar, Prado Velho, CEP 80.215-901, Curitiba/PR. DOI: http://dx.doi.org/10.18405/recfin20160302
} 
Sugerem-se estudos futuros observando outros indicadores além das bolsas de pesquisa ou analisar de modo qualitativo a essência das produções acadêmicas efetivadas por meio desse financiamento. Palavras-chave: Geração de Conhecimento; Produções Acadêmicas; Fontes de Financiamento.

\begin{abstract}
Information is a key resource within the organization, as its ability to generate innovations and best management practices. Are necessary investments to generate information and knowledge to contribute to organizations and society. In this sense, the purpose of this article is to analyze the relationship between investment of the Coordenadoria de Aperfeiçoamento de Pessoal de Nível Superior (CAPES) in the stricto sensu academic scholarships to Brazilian academic publications, from 2000 to 2012. For this was a study with exploratory and descriptive statistical analysis with the polynomial regression techniques and dispersion using secondary database. It was possible to verify empirical evidence through the coefficients of determination of statistical models. The results demonstrate that despite the CAPES data show steady increase in investment in the strict sense bags in Brazil in the period 2000 to 2012, there are no proportional increases in national scientific production in the same period. And they did not contribute effectively to the increase of the country's representation in the academic field before Latin America and the World. The final considerations highlight the key factors identified in the statistical analysis that lead to such conclusions. The study contributes to the theoretical field of studies that measure investments and innovation and helps shape applied to public resource managers. Future studies are suggest from observing other indicators in addition to research grants or analyze qualitatively the essence of academic productions effect through this funding.
\end{abstract}

Keywords: Knowledge Generation; Academic Production; Funding Sources.

\title{
1. INTRODUÇÃO
}

A geração do conhecimento é o principal objetivo da ciência. Para mensurar o desenvolvimento dos diversos campos de estudo da ciência, o acompanhamento das produções acadêmicas e sua disseminação são utilizados nessa métrica (DIAS; BARBOSA NETO; CUNHA, 2011). O conhecimento é recurso crítico nas empresas, porque é capaz de gerar inovações (AMIN; COHENDET, 2004; JOHNSON, LUNDVALL, 2000; LAM, 2000; NONAKA, TAKEUCHI, 1995; TEECE, 1998). Sendo assim, as vantagens competitivas das empresas derivam não apenas do seu market share, mas da forma como está desenvolvendo e replicando os ativos intangíveis, compreendidos neste contexto como o conhecimento da empresa. Isso faz da geração de conhecimento uma forma de enfrentar os desafios das organizações, bem como do desenvolvimento de um país.

No Brasil, a Coordenadoria de Aperfeiçoamento de Pessoal de Nível Superior (CAPES) por meio de recursos públicos - é o maior financiador de bolsas stricto sensu no País. Para Silva Filho et al. (2014 p. 5), "a função primordial da Administração Pública é empregar com eficiência os recursos públicos obtidos por meio dos tributos em favor do bem-estar social, garantindo os direitos básicos previstos na Constituição Federal de 1988".

As bolsas CAPES constituem como uma fonte relevante de financiamento acadêmico para a pesquisa. Uma das formas de analisar a eficiência desse investimento é explorar a geração de conhecimento a partir das pesquisas realizadas pelas pessoas contempladas com as bolsas. A parte objetiva da geração de conhecimento está registrada em publicações acadêmicas.

Sendo assim, o objetivo deste artigo é analisar a relação entre o investimento da Coordenadoria de Aperfeiçoamento de Pessoal de Nível Superior (CAPES) em bolsas acadêmicas stricto sensu com as publicações acadêmicas brasileiras, no período de 2000 a 2012. A análise observa o período 
de 2000 a 2012 e um total de 292.094 bolsas de mestrado no país, seguido de 198.151 bolsas concedidas para doutorado no país, em terceiro lugar 25.986 bolsas de doutorado para o exterior e por último, 211 bolsas de mestrado para o exterior.

A produção científica no Brasil neste período foi de 13.022 artigos no ano 2000 e de 53.083 artigos em 2012. É feita uma análise da base de dados Scopus e das bolsas CAPES stricto sensu concedidas. O instrumento estatístico utilizado é a regressão polinomial e os gráficos de dispersão, cujo resultado pode ser identificado através da curva de saturação e linearidade respectivamente.

O trabalho é estruturado em cinco seções. A presente introdução contextualiza a questão da fonte de financiamento acadêmico por meio de bolsas CAPES stricto sensu e a geração de conhecimento, registrada em artigos científicos. Na segunda seção está apresentado o referencial teórico sobre o tema, com enfoque na geração de conhecimento, no papel das produções acadêmicas para a geração de conhecimento e nas fontes de financiamento para a produção acadêmicas. Na terceira seção são abordados os aspectos metodológicos, especialmente o plano amostral, técnicas e instrumentos de coleta de dados. Na quarta seção são apresentadas as análises e interpretações dos resultados obtidos com o presente estudo, demonstrando as regressões polinomiais, os gráficos de dispersão, bem como a evolução dos dados de bolsas stricto sensu concedidas pela CAPES e publicações brasileiras indexadas na base Scopus durante o período de 2000 a 2012. Na quinta seção são apresentadas as considerações finais.

\section{ABORDAGEM TEÓRICA}

Neste tópico são embasados conceitos como a geração de conhecimento o papel das instituições neste sentido e fontes de financiamento que atuam na produção acadêmica com o intuito de estruturar a linha teórica da pesquisa.

\subsection{Geração de Conhecimento}

O conhecimento cada vez mais é considerado como um recurso crítico nas empresas, visto que ele é capaz de gerar inovações (AMIN; COHENDET, 2004; JOHNSON, LUNDVALL, 2000; LAM, 2000; NONAKA, TAKEUCHI, 1995; TEECE, 1998). Sendo assim, as vantagens competitivas das empresas, derivam não apenas do seu market share, mas também do desafio que existe de replicar os ativos intangíveis, compreendidos neste contexto como o conhecimento da empresa, e a forma pela qual eles estão sendo desenvolvidos. Isto quer dizer que o conhecimento deve ser criado, estocado, reproduzido, difundido e assimilado em diversas situações e deve ser transformado em valor econômico (BELUSSI, 2005).

A análise do significado do conhecimento, desde seu conceito a suas mais variadas vertentes, se faz essencial para a compreensão de sua dinâmica, tais como fontes, meios de transferência e implementação, bem como para a formulação de práticas que facilitem a sua criação, desenvolvimento e difusão. Inicialmente é preciso saber o que está sendo referido quando se trata de conhecimento, e para que seja possível saber é necessário fazer uma distinção entre o significado de dado, de informação e de conhecimento, mesmo que tais conceitos sejam complexos de serem separados. Dados podem ser definidos como "observações do estado do mundo" por Davenport e Prusak (1997) não apresentando utilidade caso nenhuma informação significativa possa ser retirada do mesmo. Os dados são importantes, mas eles são matéria-prima na criação da informação, e a interpretação da informação, traz consigo uma atualização de conhecimento gerada por meio de diferentes redes cognitivas de agentes no processo.

A informação pode ser captada por diversas formas, como por exemplo a utilização de tecnologias data mining, e se a ela for atrelado um contexto, é possível obter o conhecimento. Desta forma, a informação propicia um fluxo de mensagens, enquanto o conhecimento é criado por esse 
próprio fluxo de informação ancorado nas crenças e compromissos do seu detentor (NONAKA; TAKEUCHI, 1995).

Segundo Belussi e Pilotti (2002), a informação pode tornar-se conhecimento somente através da interpretação promovida por um agente econômico, que pode contextualizar, valorizar, categorizar, corrigir, manipular, elaborar, rearranjar, resumir e estocar partes de informações. Portanto, o conhecimento representa uma informação útil proveniente da mente humana, perpassando pelas fases de reflexão e síntese. Davenport e Prusak (2000) conseguem apresentar uma definição de conhecimento que consegue abordar seu valor, bem como sua complexidade.

Para isto, os autores definem o conhecimento como sendo uma mistura fluída de experiências, valores, informação contextual e insights que dão origem a uma estrutura para a avaliação e incorporação de novas experiências e informações (DAVENPORT; PRUSAK, 2000). Esta estrutura por sua vez é originária e ao mesmo tempo aplicada à mente dos "conhecedores". Tanto a informação quanto o conhecimento são específicos ao contexto e estão relacionados na medida em que dependem da situação e são criados de forma dinâmica a partir da interação social (NELSON; WINTER, 1982).

Um dos primeiros autores a tentar abordar o conceito de conhecimento foi Von Hayek (1945), sendo que para o autor muitas das concepções de diferentes formas de conhecimento consistem em dicotomias, principalmente devido ao fato dele não se apresentar somente na forma codificada. Ele sustenta que a ideia dos modelos econômicos formais e clássicos, os quais conduzem a um equilíbrio, ignoram uma análise da fonte de conhecimento estratégico, pois esses modelos pressupõe o fato de que todos os agentes econômicos têm conhecimento de tudo, que o conhecimento consiste num bem público e disponível a custo mínimo.

Esta visão de que o conhecimento é um bem público alterou em decorrência de diversos fatores, característicos da contemporaneidade, fazendo com que passasse a ser considerado como um bem basicamente privado, o qual apresenta altos níveis de apropriação e exclusividade.

Em seguida Von Hayek (1945) realiza uma distinção entre "conhecimento científico" e "conhecimento de uma circunstância particular de tempo e espaço", em que expressa a necessidade de se especificar os variados tipos de conhecimento, ou em outras palavras, as diferentes dimensões que o conhecimento pode adquirir em diversas circunstâncias. $\mathrm{O}$ autor assume que os indivíduos adquiram vantagens competitivas baseados na disponibilidade e uso de informações originais, além de explicar que a exclusividade está atrelada à especificidade de "tempo e espaço". Portanto, nessa abordagem, as diferenças existentes entre tecnologia e ciência são minimizadas, podendo ser dito que o fluxo tradicional está, na verdade, invertido.

De acordo com Antonelli (2005), o conhecimento científico pode ser considerado como o resultado final de um processo indutivo de articulação e codificação de conhecimento originado de forma tácita e adquirido por meio de processos de aprendizagem. Ryle (1952) aponta que o conhecimento é composto por dois fatores: know-how, o qual é a tradução pela experiência prática, habilidade, e o outro fator é o know that, que é aquela informação abstrata, tal como o conhecimento técnico. Na visão do autor, para fazer com que o know that seja útil, faz-se necessário que seja agregado a ele o know-how, apesar de o know-how não ser adquirido da mesma forma que o know that, o qual pode circular como regras e normas.

Desta forma, Ryle (1952) conclui que nem o know-how nem o know that, isolados, possuem utilidade, apenas a coexistência completa entre eles irá constituir a base de um conhecimento útil, de tal maneira que se encontra no lado oposto do conhecimento estéril, ou aquele que não possui utilidade alguma. Portanto, o conhecimento, o qual pode ser expresso em palavras e números, também chamado de conhecimento codificado, representa apenas parte do conjunto de conhecimento como um todo. Podendo concluir então que os argumentos de Ryle (1952) refutam a ideia de que a circulação de conhecimento envolve somente o conhecimento explícito. 


\subsection{Papel das Produções Acadêmicas para Geração de Conhecimento}

A ciência possui como seu principal objetivo, o fato da geração do conhecimento, considerando que a produção acadêmica e a disseminação dos resultados dessas pesquisas são impulsionadores relevantes desta expansão do saber (DIAS; BARBOSA NETO; CUNHA, 2011). A produção acadêmica, por sua vez, é um parâmetro de publicação em periódicos científicos, a qual é um dos critérios mais utilizados para a avaliação da contribuição, a evolução do conhecimento, sendo relacionada com o início, o incentivo e a consolidação de grupos de pesquisa em diversas áreas (DANTAS et al., 2011), com isto, remete-se que a produção científica de uma área de conhecimento reflete tanto o seu estado da arte, quanto sua evolução.

O conhecimento pode ser compreendido como um bem híbrido (semi-publico), pois conforme a teoria microeconômica, um bem privado puro é aquele cujo consumo é rival e exclusivo, enquanto que um bem público puro, antagonicamente, é aquele cujo consumo é não-rival e nãoexclusivo. O conhecimento, apesar de geralmente ser não-rival e não-exclusivo, pode se tornar exclusivo, como é o caso dos segredos industriais e das patentes (REIS, 2008). Seguindo pelos conceitos de Pigou (1932), é possível encontrar três mecanismos de formação do conhecimento: 1) por subsídios, 2) por pesquisa produzida diretamente pelo governo e 3) pelo "mercado do conhecimento", sendo este último item o estímulo por pesquisa e inovação gerado pelas patentes. Neste artigo, o foco é destinado a produção de conhecimento por subsídios, abordando, portanto, sobre subsídios governamentais, os quais mantêm a geração de conhecimento pelas universidades federais através de bolsas de estudo CAPES. Apesar do foco específico neste mecanismo de formação do conhecimento, é importante frisar que os outros atores potenciais na geração de conhecimento, como agências governamentais, centros de pesquisa e consultorias estão ligados às universidades e interligados entre si, formando uma rede de comunicação e pesquisa que é conduzida com interação mútua (GIBBONS et al.,1994).

A geração de novos conhecimentos nas universidades tem sido cada vez mais procurada pelas empresas e até pelo Estado, além de que a atenção dada às interações entre Estado, universidade e empresa já havia sido levantada por Sabato e Botana (1968). Na visão de Mowery e Sampat (2005), as universidades possuem papeis importantes nas economias que se apoiam no conhecimento da indústria moderna e estados de industrialização como fontes de trabalhadores treinados, com bom nível de conhecimento e ideias que fluem tanto das atividades de pesquisa mais básicas até as mais complexas.

Os produtos de importância econômica provenientes da pesquisa universitária possuem diversas formas, oscilando com o passar do tempo e de acordo com o mercado/ indústria, e buscando identificar esta importância, os autores Kim et al. (2005) registraram que existem comprovações do crescimento da influência da pesquisa universitária na inovação industrial em sua pesquisa de 1985 a 1997. Para que o ensino possa ajudar a economia e geração de inovação, as universidades fornecem a formação e o aprimoramento de profissionais, através de capacitações, de forma que possuam o conhecimento necessário para trabalhar de acordo com a demanda das empresas, sendo ainda o único local para treinamento de especialistas em número suficiente para sustentar as necessidades de áreas do conhecimento como as ciências e tecnologia (NOWOTNY et al., 2001). Dessa forma, as universidades, ao formarem pessoas qualificadas, influenciam a capacidade de absorção de conhecimentos pela sociedade, elevando a capacidade de a sociedade compreender tecnologias e conhecimentos externos, os quais não foram desenvolvidos nela, e desta forma aumentam a capacidade da sociedade utilizar esses conhecimentos. Isso permite que a sociedade seja capaz também de produzir novos conhecimentos e não apenas agir como mera copiadora ou absorvedora do que as universidades criam (NOWOTNY et al., 2001; PAVITT, 1988; ROSENBEG, NELSON, 1994).

Premus (2003) complementa ao escrever que as universidades acabam tendo impacto no crescimento econômico regional devido a excelência em pesquisa avançada e ao aumentar o contingente 
de capital humano presente na região, fazendo com que a combinação de crescimento no contingente de conhecimento e oferta de capital humano proporcione retornos crescentes na região do sistema de produção de conhecimento e na comercialização das invenções (PREMUS, 2003). Desta forma, o papel das universidades é de grande relevância como geradoras de conhecimento dentro de um sistema nacional ou regional de inovação (MAZZOLENI; NELSON, 2005; MOWERY; SAMPAT, 2005).

\subsection{Fontes de Financiamento para Produção Acadêmica}

No contexto brasileiro, o Conselho Nacional de Desenvolvimento Científico e Tecnológico (CNPq), a partir da década de 1970, passou a ter a atribuição de articulador da política de ciência e tecnologia no Brasil, com isso fico responsável por elaborar os Planos Básicos do Desenvolvimento Científico e Tecnológico (PBDCT), que passariam a fazer parte do sistema de produção científica. No caso da Capes, ela passou a ser a responsável por propor linhas de atuação em consonância com as diretrizes do Plano Nacional de Pós-graduação (PNPG) a partir de 1974. Isso por conta do Primeiro Plano Nacional de Pós-graduação que foi elaborado em 1973 no âmbito do Conselho Nacional de Pós-graduação, que vigorou até 1979 (ROMEO; ROMEO; JORGE, 2004).

Reforçando essa ideia, Schwartzman (1992) considera que hoje vemos como o apoio à pesquisa, o qual teve seu surgimento na década de 1970, com o surgimento e a consolidação das principais instituições compõem o sistema assim estruturado: o CNPq, a Capes, a Financiadora de Estudos e Projetos (FINEP) e as Fundações Estaduais de Amparo a' Pesquisa (FAPs).

O segundo PBDCT, no período compreendido entre 1980 a 1985, atribui ao CNPq a coordenação das Ações Programadas em Ciência e Tecnologia. E, a partir de 1982, a Capes passa a ser efetivamente uma agência fomentadora de programas de pós-graduação, ampliando desta forma as suas atribuições, que até então eram exclusivamente de concessão de bolsas.

Apesar de existirem diversas fontes de financiamento voltadas para o fomento da produção acadêmica, neste artigo o foco é destinado às bolsas de estudos concedidas pela CAPES para os programas de pós-graduação stricto sensu, devido sua maior representatividade na concessão de bolsas de pesquisas acadêmicas.

Ao observar dados da CAPES, é possível perceber que o número de matrículas nos cursos de pós-graduação stricto sensu no país cresceu 70\% entre 2001 e 2010, passando de 96.009 para 163.199. Esse salto foi também sustentado pelo forte investimento nacional na expansão de cursos de doutorado no país durante o período.

Acompanhando o aumento de matrículas nos cursos de pós-graduação stricto sensu houve também nesse período uma expansão no número de bolsas concedidas pela CAPES e CNPq aos alunos matriculados na pós-graduação. Segundo o CAPES, em 2001, o Brasil contava com 33.048 alunos da pós-graduação beneficiados com bolsas, o que significava $34,4 \%$ dos matriculados. Em 2010, o número de bolsas passou para 69.836 , ou seja, $42,8 \%$ dos alunos que estavam regularmente matriculados na pós-graduação stricto sensu no país. Vê-se que o crescimento na concessão de bolsas na década foi de $111,3 \%$, bem acima da taxa de crescimento do número de matrículas. Quando se analisa o número de bolsas concedidas por modalidade, constata-se que no mestrado este cresceu $129,6 \%$, e no doutorado $90 \%$.

Ao analisar o cenário paranaense durante o período 2001-2010 é possível observar os impactos da expansão do número de programas no crescimento do número total de matrículas na pósgraduação que, no período, foi de 163,9\%. Para o mestrado, o crescimento foi de $132,7 \%$, e para o doutorado 269\%. Quando se analisa a distribuição de bolsas por número de matrículas, observa-se que a expansão ocorreu junto aos novos programas de pós-graduação. Em 2001, no Paraná, 32,9\% dos alunos matriculados na pós-graduação recebiam bolsas; em 2010, eram 48\% os beneficiados. Para 2012 esse cenário se mantém. 


\section{PROCEDIMENTOS METODOLÓGICOS}

A pesquisa possui uma perspectiva epistemológica positivista com método de coleta de dados secundários e análises com tratamento estatístico. Para tanto, foram verificados os investimentos em bolsas pela CAPES, retirados da base de dados do GEOCAPES (2016), bem como os dados sobre publicações científicas no Brasil, América Latina e no Mundo que estão indexadas na base de dados Scopus, retirados da base de dados do Ministério da Ciência, Tecnologia e Inovação (MCTI).

O corte temporal dos dados foi do período 2000 até 2012, justificado pela janela de tempo disponível na base de dados GEOCAPES no ato de seleção da pesquisa. $\mathrm{Na}$ análise dos dados utilizou-se a técnica regressão polinomial e dispersão observando os indicadores de $\mathrm{R}^{2}$ para análise. Ainda com o intuito de obter maior validade e confiabilidade na pesquisa e para responder o objetivo da pesquisa, os resultados foram analisados também de modo exploratório descrevendo-os com o intuito de observar o fenômeno observado.

\section{APRESENTAÇÃO E ANÁLISE DOS RESULTADOS}

É possível verificar na tabela 1 o número de bolsas concedidas pela CAPES para programas de pós-graduação stricto sensu (mestrado e doutorado) no Brasil e no exterior. Observa-se um aumento constante de bolsas concedidas para mestrado no país, totalizando no período observado de 2000 a 2012 um total de 292.094 bolsas de mestrado no país, seguido de 198.151 bolsas concedidas para doutorado no país, em terceiro lugar 25.986 bolsas de doutorado para o exterior e por último, 211 bolsas de mestrado para o exterior.

Tabela 1 - Número de bolsas concedidas pela CAPES entre 2000-2012 para programas de pós-graduação stricto sensu (Mestrado e Doutorado) no Brasil e Exterior.

\begin{tabular}{c|c|c|c|c}
\hline Ano & $\begin{array}{c}\text { Mestrado } \\
\text { no País }\end{array}$ & $\begin{array}{c}\text { Doutorado } \\
\text { no País }\end{array}$ & $\begin{array}{c}\text { Mestrado } \\
\text { no Exterior }\end{array}$ & $\begin{array}{c}\text { Doutorado } \\
\text { no Exterior }\end{array}$ \\
\hline 2000 & 11.669 & 8.801 & 20 & 1.677 \\
\hline 2001 & 12.002 & 9.408 & 26 & 1.645 \\
\hline 2002 & 13.054 & 10.180 & 23 & 1.734 \\
\hline 2003 & 15.635 & 11.389 & 10 & 1.936 \\
\hline 2004 & 16.200 & 11.345 & 7 & 1.959 \\
\hline 2005 & 16.226 & 11.191 & 7 & 2.246 \\
\hline 2006 & 18.614 & 13.044 & 7 & 2.462 \\
\hline 2007 & 18.720 & 12.897 & 1 & 2.415 \\
\hline 2008 & 24.789 & 16.385 & 17 & 2.281 \\
\hline 2009 & 27.192 & 17.873 & 29 & 2.342 \\
\hline 2010 & 33.357 & 21.941 & 56 & 2.467 \\
\hline 2011 & 41.045 & 26.108 & - & 2.822 \\
\hline 2012 & 43.591 & 27.589 & & - \\
\hline
\end{tabular}

Fonte: adaptado de GEOCAPES (2014).

Em relação aos os dados de publicações científicas e a relevância do Brasil no campo acadêmico quando comparado a América Latina e ao Mundo, observamos na tabela 2, os dados retirados da Scopus. Trata-se da maior base de dados de resumos e citações de literatura: revistas científicas, livros e anais de congressos. Cumprindo uma visão abrangente da produção de pesquisa do mundo nas áreas de ciência, tecnologia, medicina, ciências sociais, artes e humanidades, essa base oferece ferramentas inteligentes para acompanhar, analisar e visualizar a pesquisa. 
Tabela 2 - Número de artigos brasileiros, da América Latina e do mundo publicados em periódicos científicos indexados pela Scopus, 2000-2012.

\begin{tabular}{c|c|c|c|c|c}
\hline Ano & Brasil & $\begin{array}{c}\text { América } \\
\text { Latina }\end{array}$ & Mundo & $\begin{array}{c}\text { \% do Brasil em relação à } \\
\text { América Latina }\end{array}$ & $\begin{array}{c}\text { \% do Brasil em relação } \\
\text { ao Mundo }\end{array}$ \\
\hline 2000 & 13.022 & 29.798 & 1.109 .991 & 43,7 & 1,17 \\
\hline 2001 & 14.183 & 32.135 & 1.199 .941 & 44,1 & 1,18 \\
\hline 2002 & 16.376 & 36.002 & 1.254 .870 & 45,5 & 1,3 \\
\hline 2003 & 18.455 & 40.356 & 1.322 .139 & 45,7 & 1,4 \\
\hline 2004 & 21.517 & 45.223 & 1.464 .356 & 47,6 & 1,47 \\
\hline 2005 & 24.303 & 50.798 & 1.633 .266 & 47,8 & 1,49 \\
\hline 2006 & 31.619 & 61.797 & 1.721 .655 & 51,2 & 1,84 \\
\hline 2007 & 34.005 & 65.614 & 1.811 .410 & 51,8 & 2,07 \\
\hline 2008 & 39.116 & 74.804 & 1.888 .908 & 52,3 & 2,16 \\
\hline 2009 & 42.822 & 81.745 & 1.981 .969 & 52,4 & 2,19 \\
\hline 2010 & 45.570 & 86.002 & 2.085 .164 & 53 & 2,26 \\
\hline 2011 & 49.819 & 92.760 & 2.203 .264 & 53,7 & 2,45 \\
\hline 2012 & 53.083 & 97.054 & 2.169 .154 & 54,7 & \\
\hline
\end{tabular}

Fonte: Ministério da Ciência, Tecnologia e Inovação (MCTI).

É possível observar na tabela 2 um crescimento significante referente a representatividade do Brasil em relação a América Latina, passando de 47,8\% em 2005 para 52,5 em 2008. No mesmo período, houve um crescimento de 1,49\% para 2,07 referentes à representatividade das publicações brasileiras em relação ao mundo.

Figura 1 - Regressão polinomial bolsas stricto sensu.

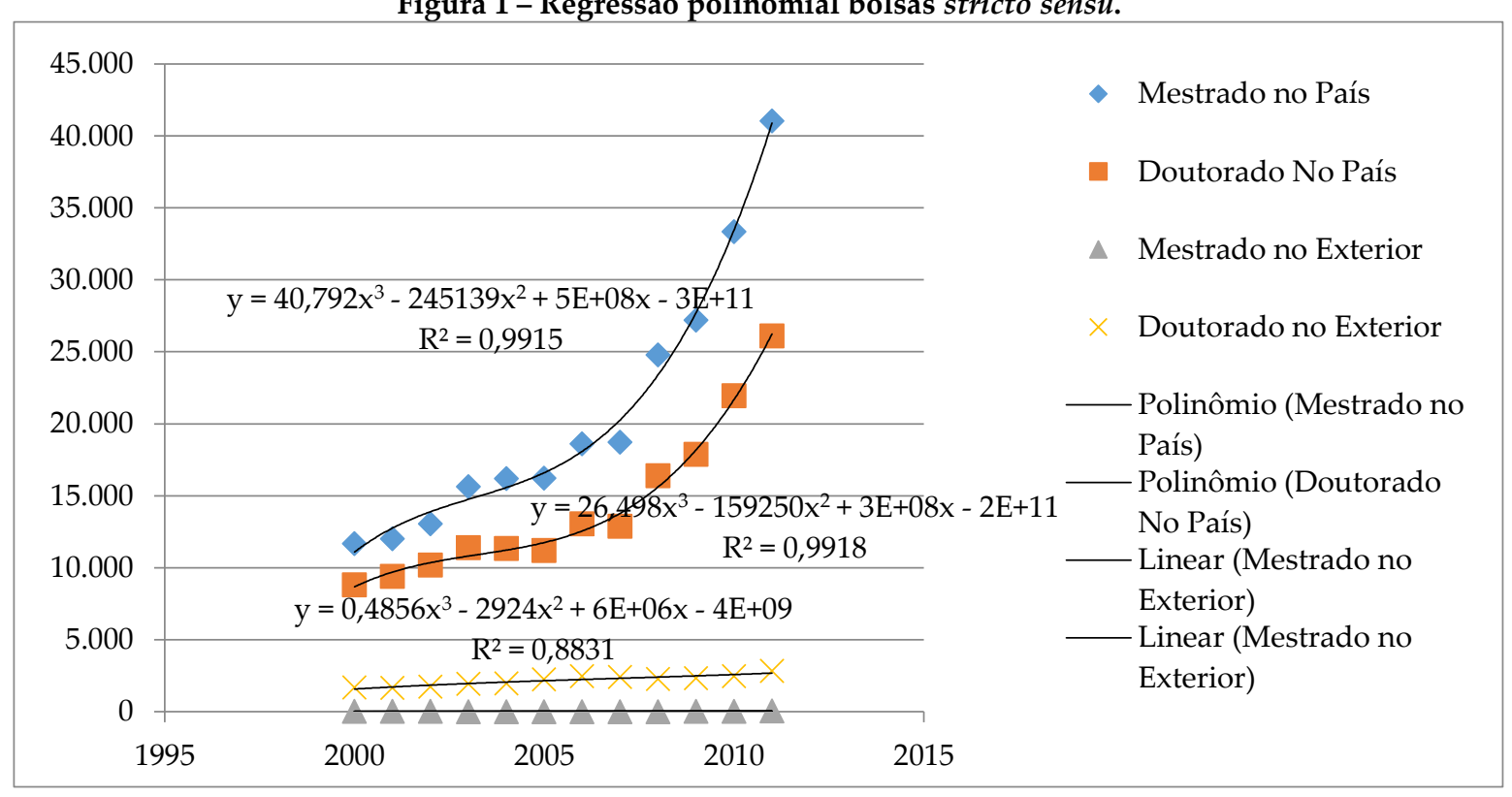

Fonte: elaborado pelos autores.

Foi realizada uma regressão polinomial conforme a figura 1 de ordem três para observar a relação entre as variáveis de tempo "n" (2000 a 2011) e concessão de bolsas neste mesmo período. Observamos principalmente pelo $\mathrm{R}^{2}$ das bolsas de mestrado no país no valor de 0,9915 e bolsas de doutorado no país no valor de 0,9918 que há um aumento real das bolsas concedidas em stricto sensu no período. Podemos verificar por meio do valor de beta, identificado pelo "Y", o aumento da média anual de concessão de bolsas por modalidade (mestrado e doutorado no país ou internacional). 
Nesse sentido, é possível observar um crescimento constante principalmente no investimento em bolsas de mestrado e bolsas de doutorado no país.

Na sequência da apresentação dos dados, observa-se, por meio de um gráfico de linhas, a evolução no número total de bolsas concedidas pela CAPES para pós-graduação stricto sensu, contemplando mestrado e doutorado no país, além de mestrado e doutorado internacional. Além das publicações em periódicos nacionais indexados na base de dados Scopus no período de 2000 a 2011.

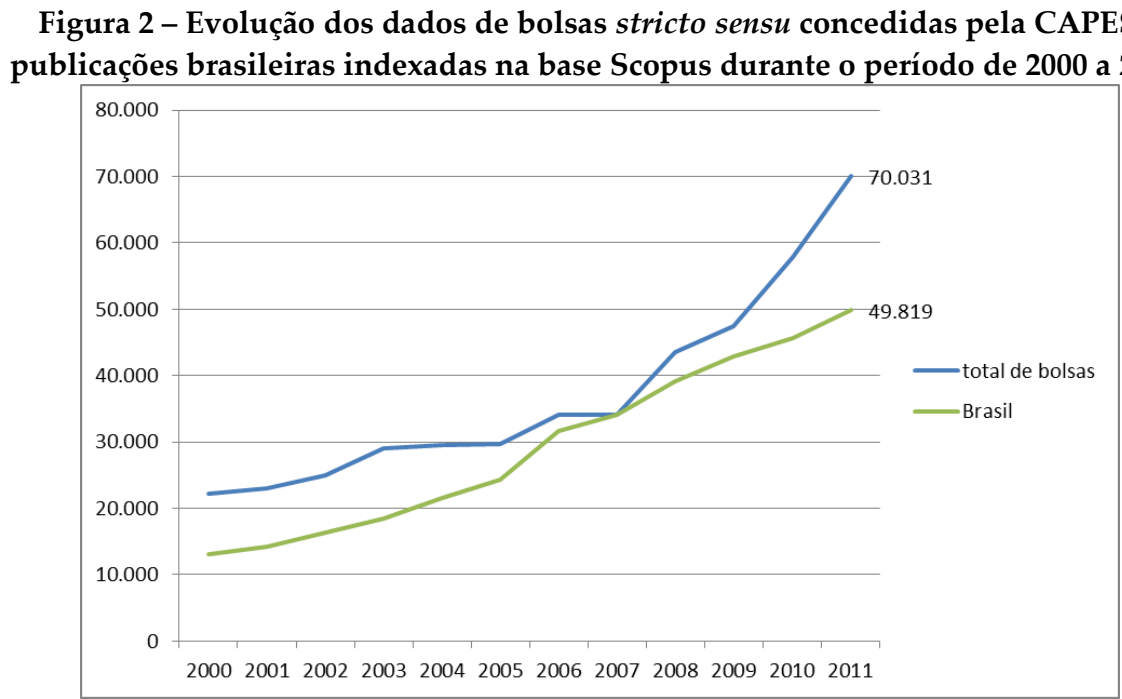

Fonte: elaborado pelos autores.

Observa-se na figura 2, no período entre 2006 a 2008 um crescimento significante nas bolsas concedidas para as pesquisas stricto sensu, crescimento este, que não é acompanhando na mesma proporção pelas publicações científicas no Brasil. Inferindo por meio do gráfico, que o investimento em bolsas stricto sensu não contribui de maneira efetiva para o aumento de publicações no Brasil.

Para complementar a análise anterior, realizamos uma regressão polinomial exposta na figura 3 para observar o comportamento das variáveis "investimentos em bolsas stricto sensu" e "publicações em periódicos no Brasil” no período de 2000 a 2011.

Figura 3 - Regressão polinomial sobre Investimentos em bolsas stricto sensu e publicações 2000 - 2011.

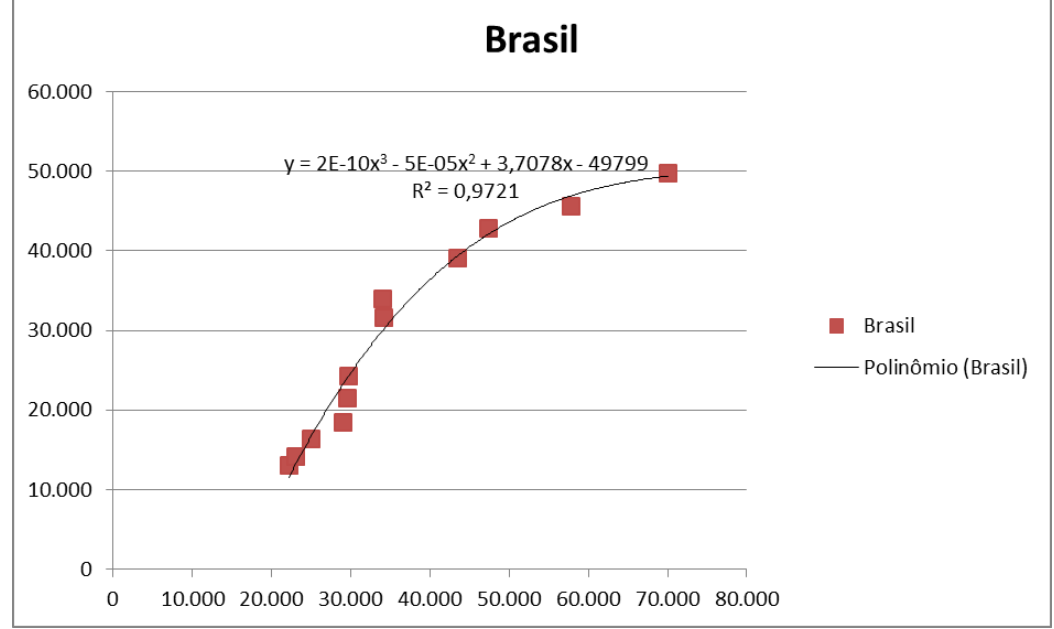

Fonte: elaborado pelos autores.

A análise longitudinal, retrospectiva, com os dados de publicações nacionais através de um levantamento na base de dados SCOPUS. Os dados obtidos foram submetidos à análise de variância com o fator desdobrado em parâmetros de regressão polinomial. 
Para que uma curva ou função de ajuste seja considerada boa, os pontos experimentais não devem estar muito afastados dos pontos calculados, como também pode-se desconfiar daqueles que estão absolutamente em concordância com os pontos calculados. Os critérios de avaliação da qualidade do ajuste buscam determinar o grau de verossimilhança da curva ajustada em relação aos pontos experimentais (VUOLO, 1998).

Neste estudo foram aplicados os ajustes de ordem 3, demonstrando uma saturação na correlação de investimentos em bolsas com o resultado de publicações, fazendo com que o impacto decorrente de maiores investimentos em bolsas seja cada vez menor no aumento de produções acadêmicas. O gráfico está caminhando para uma saturação. Esta indicação pode inferir que os investimentos em bolsas não contribuirão diretamente positivamente para o crescimento das publicações Brasileiras. Nesse contexto, a tabela 3 demonstra os dados consolidados do total de bolsas stricto sensu concedidas pela CAPES por período, bem como a alteração da representatividade das publicações brasileiras em relação a América Latina e ao Mundo.

Tabela 3 - Total de bolsas pelo período e alteração da representatividade do Brasil em relação a América Latina e ao Mundo.

\begin{tabular}{c|c|c|c}
\hline Período & Total de Bolsas & $\begin{array}{c}\text { \% do Brasil em relação à } \\
\text { América Latina }\end{array}$ & $\begin{array}{c}\text { do Brasil em relação ao } \\
\text { Mundo }\end{array}$ \\
\hline $2000-2001$ & 914 & 0,4 & 0,01 \\
\hline $2001-2002$ & 1910 & 1,4 & 0,12 \\
\hline $2002-2003$ & 3977 & 0,2 & 0,1 \\
\hline $2003-2004$ & 546 & 1,9 & 0,02 \\
\hline $2004-2005$ & 156 & 0,2 & 0,35 \\
\hline $2005-2006$ & 4457 & 3,4 & 0,04 \\
\hline $2006-2007$ & -88 & 0,6 & 0,19 \\
\hline $2007-2008$ & 9417 & 0,5 & 0,09 \\
\hline $2008-2009$ & 3968 & 0,1 & 0,03 \\
\hline $2010-2011$ & 10370 & 0,6 & 0,07 \\
\hline
\end{tabular}

Fonte: elaborado pelos autores.

Figura 4 - Gráfico da representatividade do Brasil em relação à América Latina e ao Mundo.

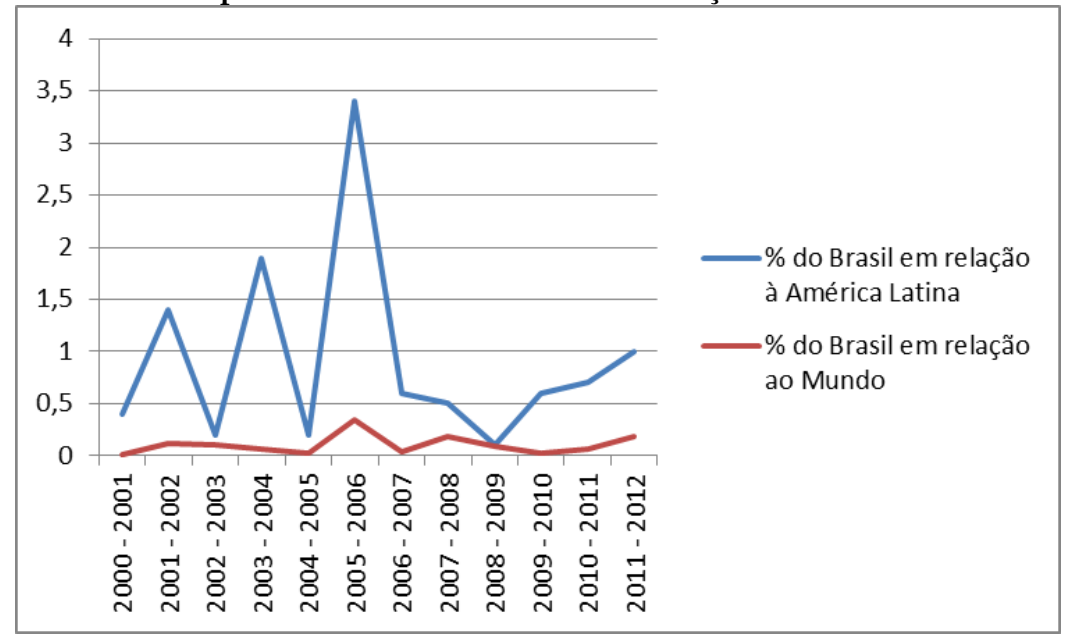

Fonte: elaborado pelos autores. 
É possível observar na figura 4 um aumento significativo na representatividade do Brasil em relação à América Latina e em relação ao mundo no período de 2005 a 2008, contudo, ao analisar o crescimento do nível de publicações no Brasil, não é possível justificar o aumento da representatividade. Visto que, como analisamos anteriormente, o aumento das publicações em periódicos nacionais não acompanhou o crescimento de investimento em bolsas de pós-graduação stricto sensu o período.

Para observar uma possível relação entre o aumento de bolsas concedidas e a melhoria na representatividade do Brasil em relação à América Latina e ao Mundo, observou-se por meio de gráficos de dispersão a alteração anual da representatividade do Brasil em relação ao número de bolsas stricto Sensu concedidas pela CAPES durante o período de 2000 a 2011.

Figura 5 - Representatividade do Brasil em relação a América Latina e a representatividade do Brasil em relação ao mundo.

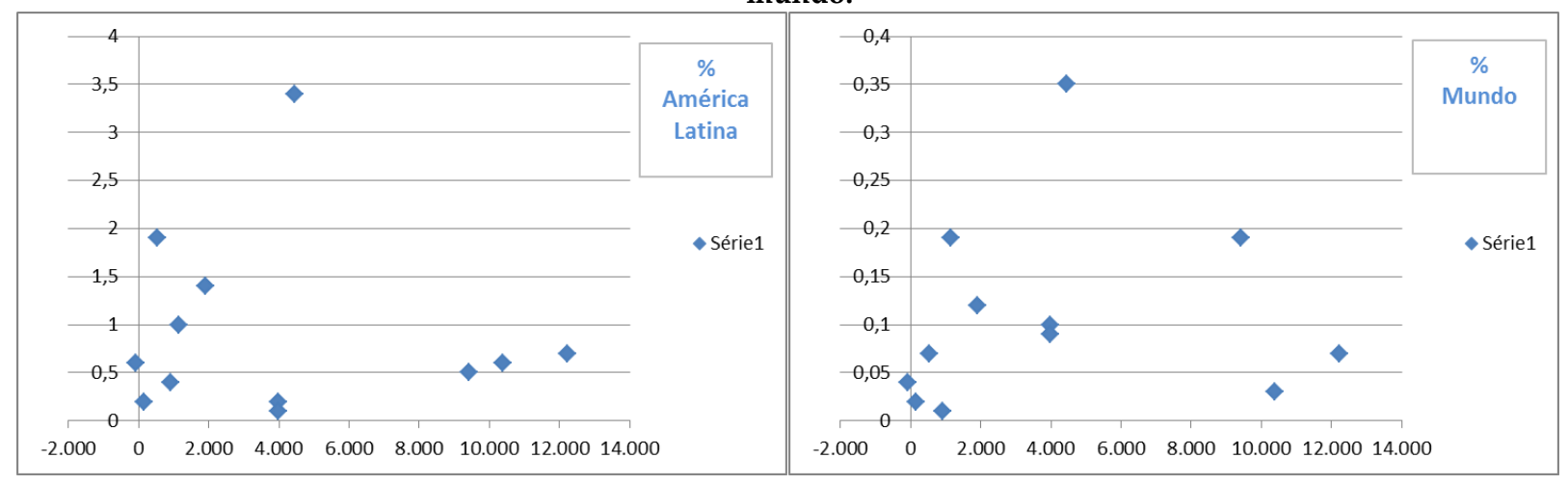

Fonte: Elaborado pelos autores.

Pelas representações gráficas de distorções observadas na figura 5, é possível verificar que não se consolida nenhum padrão de comportamento que possa inferir alguma relação de comportamento entre o $\mathrm{n}^{\mathrm{o}}$ de bolsas stricto sensu concedidas e o comportamento da representatividade do Brasil.

Em resposta a esse fenômeno, recorreu-se a literatura para compreendê-lo. Diante disso, a Agência Brasileira de Inteligência (ABIN, 2014), afirma que as produções científicas de determinadas áreas de conhecimento, destacando temáticas como: agropecuária, exportação, automação bancária, votação eletrônica, aviação, mineração, biocombustíveis, tecnologia do pré-sal, entre outros, despertaram interesse internacional. Fruto disso, entre o período de 2007 e 2008 houve uma significativa ampliação do número de periódicos nacionais indexados na base Web of Science.

Uma característica relevante, é o fato de que ao ser indexado na base, todo o histórico do periódico é adicionado, assim, números de períodos anteriores podem sofrer alterações, como visualizados no índice de representatividade do Brasil em relação à América Latina e ao Mundo nos anos de 2005 e 2006. Reconhecida mundialmente como referência a Web of Science, originalmente (Institute for Scientific Information - ISI), foi à primeira base de dados a catalogar as revistas científicas mais importantes do mundo.

Tabela 4 - Base de dados da Web of Science (Portfólio).

\begin{tabular}{l|l}
\hline Portfólio & Revistas Científicas (Journal) \\
\hline$+20 \mathrm{mil}$ & Livros Escolares \\
\hline$+60 \mathrm{mil}$ & Conferências realizadas anualmente \\
\hline$+15 \mathrm{mil}$ & Patentes \\
\hline+51 milhões
\end{tabular}

Fonte: Adaptado Web of Science. 
Atualmente, a representatividade da América Latina nos indexados da Web of Science é de $2 \%$, mesma representatividade de Oriente Médio em conjunto com África. Adiante, o continente asiático com $8 \%$, América do Norte com $40 \%$ e finalmente, o continente europeu com $48 \%$. Em relação às áreas de conhecimento contempladas pelo portfólio, $10 \%$ são sob a perspectiva de Artes \& Humanas, 21\% Ciências Sociais e Ciências Naturais com 69\% do portfólio.

Segundo a ABIN (2014), "o número de publicações brasileiras indexadas na base de dados da Web of Science, que pertence à empresa canadense Thomson Reuters, quadruplicou em três anos. Era de 26, em 2006; passou para 63, em 2007; e chegou a 103, em 2008". Diante disso, é possível inferir que não houve nesse período de 2006 a 2008, um crescimento real, como se demonstra nos gráficos.

\section{CONSIDERAÇÕES FINAIS}

Para Pigou (1932), um dos mecanismos possíveis de formação de conhecimento é por meio de subsídios. Nesse sentido, foi analisada a relação entre o investimento da Coordenadoria de Aperfeiçoamento de Pessoal de Nível Superior (CAPES) em bolsas acadêmicas stricto sensu com as publicações acadêmicas brasileiras, no período de 2000 a 2012.

Diferentemente do que se esperava, os resultados obtidos por meio de análises estatísticas descritivas não demonstraram grande contribuição dos investimentos em bolsas stricto sensu em relação ao aumento da produção acadêmica no Brasil. O gráfico de regressão polinomial de ordem três demonstra uma visível saturação entre as variáveis, onde é possível inferir, que a efetividade dos investimentos realizados no período de 2000 a 2012 no sentido de contribuir para o aumento das publicações estão perdendo força.

Em relação a representatividade do campo acadêmico Brasileiro em relação à América Latina e em relação ao Mundo, observamos principalmente no período de 2006 a 2008, um significante aumento como abordado na seção 4, porém análises estatísticas descritivas de dispersão demostraram que não há um comportamento que estabeleça qualquer relação entre os investimentos realizados pela CAPES no período de 2000 a 2012 com o aumento da representatividade do Brasil no campo acadêmico.

Visto isto, a literatura apresenta uma justificativa de que o aumento da representatividade não infere um aumento real na produção acadêmica do Brasil em relação à América Latina e ao Mundo, visto que tal aumento se deve ao fato de que no período de 2007 a 2008, vários periódicos nacionais foram indexados na base de dados internacional Web Of Science. Este fato foi o grande propulsionador do aumento da representatividade das produções acadêmicas brasileiras.

Diante desse cenário, os autores sugerem que mais pesquisas sejam realizadas abrangendo o fenômeno das produções científicas, com vistas a compreender quais são os principais motivadores e como contribuem para o fenômeno. Em tempo, os autores defendem que muito embora, neste estudo, tenha se demonstrado que os investimentos em bolsas stricto sensu realizados pela CAPES não obtiveram grande efetividade no aumento das publicações nacionais no período de 2000 a 2012, os investimentos são fundamentais para a manutenção e contribuições do campo acadêmico brasileiro para o mundo.

\section{REFERÊNCIAS}

ABIN. Agência Brasileira de Inteligência. Brasil avança no ranking da produção científica mundial. 2009. Disponível em: http://www.abin.gov.br/modules/articles/article.php?id=4516. Acesso em: $10 \mathrm{dez}$. 2015.

AMIN, A.; COHENDET, P. Architectures of Knowledge. Firms, Capabilities and Communities. Oxford: Oxford University Press, 2004. 
ANTONELLI, C. Models of Knowledge and Systems of Governance. Journal of Institutional Economics, v. 1, n. 1, p. 51-73, 2005.

BELUSSI, F.; PILOTTI, L. Knowledge Creation, Learning and Innovation in Italian Industrial Districts. Geografiska Annaler, v. 84, p. 125-139, 2002.

BELUSSI, F. Industrial Districts/local production systems as hyper-networks: a neo- marshallian interpretative frame. IN: The changing Firm Contributions from the History of Economic Thought. Guidi \& Parisi (eds.), 1a. ed, Franco Angeli, Milano, 2005.

DANTAS, J. A. et al. Padrões de comunicação científica em contabilidade: um comparativo entre a Revista Contabilidade e Finanças e a The Accounting Review. Revista Contemporañea de Contabilidade, v. 8, n. 16, p. 11-36. 2011.

DAVENPORT, H.; PRUSAK, L. Information Ecology: Mastering the information and Knowledge Environment, Oxford University Press, 1997.

DAVENPORT, H.; PRUSAK, L. Working Knowledge: How Organizations Manage What They Know, Harvard Business School Press, 2000.

DIAS, W. O.; BARBOSA NETO, J. E.; CUNHA, J. V. A. A comunicação do conhecimento científico: dados sobre a celeridade do processo de avaliação e de publicação de artigos científicos em periódicos da área de contabilidade. Revista Contemporañea de Contabilidade, v. 8, n. 15, p. 41-62, 2011.

GEOCAPES. Sistema de Informações Georreferenciadas - CAPES - Disponível em: http://geocapes.capes.gov.br/geocapes2. Acesso em: 20 de set. 2016.

GIBBONS. M. et al. The New Production of Knowledge: The Dynamics of Science and Research in Contemporary Societies. SAGE. London. 1994.

JOHNSON, B.; LUNDVALL, B. Promoting Innovation Systems as a Response to the Globalizing Learning Economy. In: Cassiolato, J et al. Arranjos e Sistemas Produtivos Locais e as Novas Politicas de Desenvolvimento Industrial e Tecnológico. Rio de Janeiro: IE/UFRJ. Contrato BNDES/FINEP-FUJB, 2000.

LAM, A. Tacit Knowledge, Organizational Learning and Societal Institutions: An integrated Framework. Organizational Studies, v. 21, n. 3, p. 487-513, 2000.

KIM, J.; LEE, S. J.; MARSCHKE, G. The Influence of University Research on Industrial Innovation, NBER - National Bureau of Economic Research, v. 11447, 2005.

MATTOS, E. J.; BAGOLIN, I. P. Desenvolvimento econômico no Rio Grande do Sul: já não somos quem éramos? Porto Alegre: EDIPUCRS, 2014.

MAZZOLENI, R; NELSON, R. The Roles of Research at Universities and Public Labs in Economic Catch-up. Research Policy, v. 36, p. 1512-1528, 2007.

MINISTÉRIO DA CIÊNCIA, TECNOLOGIA E INFORMAÇÃO. Número de artigos brasileiros, da América Latina e do mundo publicados em periódicos científicos indexados pela Thomson/ISI e 
Scopus, 1996-2012. Disponível em: http://www.mct.gov.br/index.php/content/view/5710.html. Acesso em: 01 dez. 2015.

MOWERY. D.; SAMPAT. B. Universities in National Innovation Systems. In: FAGERBERG. J.; MOWERY. D.; NELSON. R. The Oxford Handbook of Innovation. Oxford: University Press. 2005.

NELSON, R.; WINTER, S. An Evolutionary Theory of Economic Change. Cambridge: Harvard University Press, 1982.

NONAKA, I; TAKEUCHI, H. The Knowledge-creating Company. New York: Oxford Univ. Press, 1995.

NOWOTNY. H. et al. Re-Thinking Science: Knowledge and the Public in an Age of Uncertainty. Polity Press: Cambridge, 2001.

PAVITT, K. International patterns of technological accumulation. In HOOD, N.; VAHLNE, J. E. (eds) International patterns of technological accumulation, London: Croom Helm, 1988.

PIGOU, A.C. The Economics of Welfare, London: Macmillan, 4 ed, 1932.

PREMUS. R. University knowledge production and industrial innovation: the evidence. International Journal of Technology Transfer and Commercialisation, v. 2, n. 3, p. 263-273, 2003.

REIS, D. R. Gestão da inovação tecnológica. Barueri. SP: Ed. Manole, 2008.

ROMEO, J. R. M; ROMEO, C. I. M; JORGE, V. L. Estudos de pós-graduação no Brasil. Rio de Janeiro: UNESCO, 2004. Disponível em: http://nucleodememoria.vrac.puc-rio.br/site/textosfinais/romeo2004.pdf. Acesso em: 06 dez. 2015.

RYLE, G. The Concept of Mind. London: Hutchinson University Library, 1949.

SABATO J. A.; BOTANA, N. La ciencia y la tecnología en el desarrollo futuro de América. Latina. Revista de la Integración, n. 3, p. 15-36, 1968.

SCHWARTZMAN, S. O apoio à pesquisa no Brasil. Interciência. Caracas, v. 17, n. 11, p. 329-333, 1992.

SILVA FILHO et al. Análise da eficiência nos gastos públicos com educação fundamental nos colégios militares do exército: evidência para os anos de 2009 e 2011. In: XIV Congresso USP Controladoria e Contabilidade. Anais ... São Paulo, 2014.

TEECE, D. Capturing Value from Knowledge Assets: The new economy, markets for know-how, and intangible assets. California Management Review, v. 40, n. 3, 1998.

WEB OF SCIENCE. The Citation Conection. [2014]. Disponível em: http://wokinfo.com/citationconnection/popup.html. Acesso em: 10 dez. 2015.

VON HAYEK, F. The Use of Knowledge in Society. The American Economic Review, v. 35, n. 4, p. 519530, 1945.

VUOLO, J.H. Fundamentos da Teoria de Erros. Editora Edgard Blücher: São Paulo, 1998. 